\title{
Effectiveness and Comparison of Mulligan Techniques in Improving Straight Leg Raise in Subjects with Hamstring Tightness
}

\author{
Aqsa Mobeen ${ }^{1}$, Maham Javed ${ }^{2}$, Muhammad Umer ${ }^{3}$, Jibran Arshad ${ }^{1}$, Abdul Ghafoor Sajjad ${ }^{3}$ and \\ Mubin Mustafa Kiyani*4 \\ ${ }^{1}$ Faculty of Rehabilitation and Allied Health Sciences, Islamabad, Pakistan \\ ${ }^{2}$ University of Lahore Islamabad Campus, Islamabad, Pakistan
}

${ }^{3}$ Department of Rehabilitation Sciences, Islamabad, Pakistan

${ }^{4}$ Shifa College of Medical Technology, Islamabad, Pakistan

*Corresponding author: Mubin Mustafa Kiyani, Shifa College of Medical Technology, Islamabad, Pakistan

\section{ARTICLE INFO}

Received: 蔧 January 21, 2021

Published: 幽 January 29, 2021

Citation: Aqsa Mobeen, Maham Javed, Muhammad Umer, Jibran Arshad, Abdul Ghafoor Sajjad, Mubin Mustafa Kiyani. Effectiveness and Comparison of Mulligan Techniques in Improving Straight Leg Raise in Subjects with Hamstring Tightness. Biomed J Sci \& Tech Res 33(3)2021. BJSTR. MS.ID.005417.

Keywords: Hamstring Muscles; Range of Motion; Traction; Pain; Manipulation; Mulligan

\section{ABSTRACT}

To evaluate the effectiveness of Mulligan bent leg raise technique, 2 legs rotation technique and straight leg raise with traction technique in subjects with hamstring tightness. 64 normal healthy subjects ( 25 females and 39 males) were recruited in the study under simple randomization method. Purposive nonprobability convenient sampling technique was used to collect the data. Group A received single session of Mulligan bent leg raise technique, Group B received single session of Mulligan two legs rotation technique and group $\mathrm{C}$ received single session of Mulligan straight leg raise with traction technique for hamstring tightness. Numeric pain rating scale and straight leg raise range were measured pre intervention and post intervention. Data was analyzed by IBM SPSS 21 using paired t-test and one-way anova. Numeric Pain Rating Scale was significantly reduced in all 3 groups with a $p$ value $p<0.01$ and Straight leg raise was significantly improved post single session of intervention with $p$ value $p<0.01$. All Mulligan techniques were effective in improving pain score and straight leg raise range of motion but bent leg raise technique was most effective among all.

\section{Introduction}

Flexibility is an attribute that enhance optimal physical functioning and is defined as the ability of an individual to move smoothly. Our body has multiple muscle groups, some of them have tendency to get weak and some muscle groups are prone to tightness. Muscle tightness is caused by decrease in flexibility of muscle and is assumed as an intrinsic risk factor for causing muscle injury resulting in reduced range of motion of the respected joint Hamstring is one of the muscle groups that is prone to tightness Hamstring muscles cover the posterior aspect of thigh and consists of semimembranosus, semitendinosus and biceps femoris. Inability to achieve more than 160 degrees range of knee extension with 90 degrees of hip flexion is deliberated as hamstring tightness. Hamstring injuries and strains are common in athletes and are caused by lack of hamstring flexibility. These injuries are slowly recovered and decrease the overall performance or normal individuals as well as athletes [1]. One of the causes of low back pain is hamstring tightness. Recent studies suggest that subjects who perceive their hamstrings to be tight are unlikely to have altered hamstring muscle extensibility or length. Altered neural tissue mobility also referred to as altered neurodynamic could be a significant contributor to "perceived hamstrings tightness". Conventionally, hamstrings stretching exercises are employed to treat perceived hamstrings tightness. There is a paucity of literature assessing the effectiveness of exercises targeting neural tissue 
mobility or neurodynamic as opposed to conventional hamstrings stretching exercises [2].

Conventional treatment and stretching techniques are used most to increase hamstrings flexibility. A study was conducted on the title of evaluation of intra-subject difference in hamstring flexibility in patients with low back pain by Radwan Ahmed et al. in January 2015. Participants included in this study with mechanical low back pain were 72 and it was concluded that hamstring tightness and mechanical low back pain have a possible relationship among them. Severity of low back pain was more evident among participants with hamstring tightness. Unilateral non dominant lower extremity hamstring tightness was more observed in patients with mechanical low back pain [3]. A study was done by David O Draper et al. on the title of Shortwave Diathermy and Prolonged Stretching Increase Hamstring Flexibility More Than Prolonged Stretching Alone. 30 college students with hamstring tightness were recruited in study. It was observed that hamstring flexibility was increased more with the use of shortwave diathermy in conjunction with prolonged hamstring stretching [4].

A Randomized Controlled Trial of Hamstring Stretching: Comparison of Four Techniques was conducted by Fasen Jo $\mathrm{M}$ et al. 100 participants were included in study. The efficacy of Active stretching, passive stretching, neural mobilization and proprioceptive neuromuscular facilitation techniques was observed. Active stretching significantly improved hamstring length during initial 4 weeks of stretching. Hamstrings length for active stretching group decreased from 4 to 8 weeks. After 8 weeks passive stretching had greatest improvement in hamstring length. PNF technique in 90/90 active stretch showed more improvement in knee range of motion than 90/90 passive techniques. No correlation was observed between hamstring flexibility and initial tightness, exercises frequency per week and age [5]. Emilio J Puentedura et al. conducted a study on immediate effects of quantified hamstring stretching: Hold-relax proprioceptive neuromuscular facilitation versus static stretching. 33 subjects with hamstring shortness were recruited in study. Comparing the effectiveness of HR- PNF and SS techniques showed no significant difference. Both stretching techniques resulted in significant immediate increase in hamstring flexibility [6].

\section{Objective of the Study}

To compare and evaluate the effectiveness of Mulligan's bent leg raise technique, two legs rotation and leg raise with traction to improve Straight leg raise in subjects with hamstring tightness.

\section{Materials and Methodology}

The study was conducted at Pakistan railway hospital, Rawalpindi from March 15, 2018 to May 23, 2018. Study design was randomized control trial.64 normal healthy subjects (25 females and 39 males) were recruited in the study. Purposive nonprobability convenient sampling technique was used to collect the data. Normal healthy subjects of age 21 to 80 years with minimum 20 degrees of restriction in Straight leg raise (SLR) unilaterally were included in the study. Subjects with lower extremity injuries, neurological problems and involved in sports activities were excluded from the study. The duration of study was 2 months. Subjects were allocated randomly into 3 groups by using coin and toss method. Group A (Mulligan bent leg raise technique), Group B (Mulligan two legs rotation technique) and group $\mathrm{C}$ (straight leg raise with traction technique). Subjects received only single session of Mulligan techniques. Self-structured questionnaire was used to collect the data. Pain before and after application of technique was measured by numeric pain rating scale (NPRS) and range of motion for straight leg raise was measured by goniometer pre- and post-intervention. Informed consent was taken from subjects before data collection and ethical considerations were followed. Data was managed and analyzed statistically by using IBM SPSS software version 21 . Paired t- test was applied within the groups to test whether there was reduction in pain and improvement in ROM, post- intervention. One-way Anova test was applied to make comparison between groups. Results were displayed in the form of tables.

\section{Results}

Table 1: Demographics.

\begin{tabular}{|c|c|c|c|}
\hline Variable & Overall & Group 1 & Group 2 \\
\hline Age & Mean: $41.5 \pm 16.09$ & Mean: $43.4 \pm 15.7$ & Mean: $45.04 \pm 18.2$ \\
\hline Gender & M (25), F (39) & M (10), F (15) & M (9), F (12) \\
\hline Referral diagnosis & Low back ache: $22(34.4 \%)$ & $\begin{array}{c}\text { Lumbar spondylosis: } \\
8(32 \%)\end{array}$ & Low back ache: $6(28.6 \%)$ \\
\hline Re-diagnosis: & Limited SLR: $30(46.9 \%)$ & Limited SLR: $14(56 \%)$ & Limited SLR: $10(47.6 \%)$ \\
\hline LPRS before technique & $5.76 \pm 1.90$ & $5.72 \pm 1.45$ & $6.09 \pm 2.11$ \\
\hline NPRS after technique & $4.07 \pm 1.68$ & $3.80 \pm 1.41$ & $4.71 \pm 1.64$ \\
\hline SLR before technique & $39.95 \pm 16.43$ & $38.6 \pm 13.4$ & $40.90 \pm 17.82$ \\
\hline SLR after technique & $53.18 \pm 19.36$ & $53.4 \pm 18.1$ & $5.72 \pm 1.93$ \\
\hline
\end{tabular}


Among 64 subjects, there were 25 males and 39 females. Mean age of subjects was $41.5 \pm 16.09$. Majority of subjects 22 (34.4\%) were observed with referral diagnosis of low back ache. Re-diagnosis of majority of subjects 30 (46.9\%) was limited SLR. NPRS before technique was $5.76 \pm 1.90$ and after technique was $4.07 \pm 1.68$. SLR before technique was $39.95 \pm 16.43$ and after technique was 53.18 \pm 19.36. (Table 1) Paired t test was applied within groups to show pre- and post-intervention difference. NPRS and SLR were significantly improved within all 3 groups with a p value $<0.05$ (Table 2). An analysis of variance showed that NPRS score before technique between groups and within groups was non-significant with a p-value i.e., $p>0.05, F(2,61)=0.572, p=0.567$. NPRS score after technique between groups and within groups was non-significant with a p-value i.e., $p>0.05, \mathrm{~F}(2,61)=2.337, \mathrm{p}=0.105$. (Table 3 )

Table 2: Paired T-Test.

\begin{tabular}{|c|c|c|c|c|c|}
\hline & Test variables & Pre mean \pm SD & Post mean \pm SD & Mean difference & P- value \\
\hline \multirow{2}{*}{ Group 1 } & NPRS & $5.72 \pm 1.45$ & $3.80 \pm 1.41$ & $1.92 \pm 0.04$ & $P<0.01(0.000)$ \\
\cline { 2 - 6 } & SLR & $38.68 \pm 13.40$ & $53.40 \pm 18.10$ & $14.72 \pm 4.7$ & $P<0.01(0.000)$ \\
\hline \multirow{2}{*}{ Group 2 } & NPRS & $6.09 \pm 2.119$ & $4.71 \pm 1.64$ & $1.38 \pm 0.47$ & $P<0.01(0.000)$ \\
\cline { 2 - 6 } & SLR & $40.90 \pm 17.8$ & $53.33 \pm 20.75$ & $12.43 \pm 2.95$ & $P<0.01(0.000)$ \\
\hline \multirow{2}{*}{ Group 3 } & NPRS & $5.44 \pm 2.20$ & $3.72 \pm 1.93$ & $1.72 \pm 0.27$ & $P<0.01(0.000)$ \\
\cline { 2 - 6 } & SLR & $40.61 \pm 19.22$ & $52.72 \pm 20.48$ & $12.11 \pm 1.26$ & $P<0.01(0.000)$ \\
\hline
\end{tabular}

Table 3: One way ANOVA.

\begin{tabular}{|c|c|c|c|c|}
\hline & & Degree of freedom & $\mathbf{F}$ & p-value \\
\hline \multirow{2}{*}{ NPRS before technique } & Between groups & 2 & \multirow{2}{*}{0.572} & \multirow{2}{*}{0.567} \\
\hline & Within groups & 61 & & \\
\hline \multirow{2}{*}{ NPRS after technique } & Between groups & 2 & \multirow{2}{*}{2.337} & \multirow{2}{*}{0.105} \\
\hline & Within groups & 61 & & \\
\hline \multirow{2}{*}{ SLR before technique } & Between groups & 2 & \multirow{2}{*}{0.121} & \multirow{2}{*}{0.886} \\
\hline & Within groups & 61 & & \\
\hline \multirow{2}{*}{ SLR after technique } & Between groups & 2 & \multirow{2}{*}{0.007} & \multirow{2}{*}{0.993} \\
\hline & Within groups & 61 & & \\
\hline
\end{tabular}

An analysis of variance showed that SLR before technique before technique between groups and within groups was non-significant with a p-value i.e., $p>0.05, \mathrm{~F}(2,61)=0.121, \mathrm{p}=0.886$. SLR after technique between groups and within groups was non-significant with a p-value i.e., $p>0.05, F(2,61)=0.007, p=0.993$ (Table 3). A

Table 4: Post hoc Tukey.

\begin{tabular}{|c|c|c|c|}
\hline \multirow{6}{*}{ NPRS before technique } & \multirow{2}{*}{ Group A } & Group B & $\mathrm{P}=0.786$ \\
\hline & & Group C & $\mathrm{P}=0.887$ \\
\hline & \multirow{2}{*}{ Group B } & Group A & $\mathrm{P}=0.786$ \\
\hline & & Group C & $P=0.543$ \\
\hline & \multirow{2}{*}{ Group C } & Group A & $\mathrm{P}=0.887$ \\
\hline & & Group B & $P=0.543$ \\
\hline \multirow{6}{*}{ NPRS after technique } & \multirow{2}{*}{ Group A } & Group B & $\mathrm{P}=0.155$ \\
\hline & & Group C & $\mathrm{P}=0.987$ \\
\hline & \multirow{2}{*}{ Group B } & Group A & $\mathrm{P}=0.155$ \\
\hline & & Group C & $\mathrm{P}=0.155$ \\
\hline & \multirow{2}{*}{ Group C } & Group A & $P=0.987$ \\
\hline & & Group B & $\mathrm{P}=0.155$ \\
\hline
\end{tabular}




\begin{tabular}{|c|c|c|c|}
\hline \multirow{6}{*}{ SLR before technique } & \multirow{2}{*}{ Group A } & Group B & $\mathrm{P}=0.894$ \\
\hline & & Group C & $\mathrm{P}=0.926$ \\
\hline & \multirow{2}{*}{ Group B } & Group A & $\mathrm{P}=0.894$ \\
\hline & & Group C & $\mathrm{P}=0.998$ \\
\hline & \multirow{2}{*}{ Group C } & Group A & $\mathrm{P}=0.926$ \\
\hline & & Group B & $\mathrm{P}=0.998$ \\
\hline \multirow{6}{*}{ SLR after technique } & \multirow{2}{*}{ Group A } & Group B & $\mathrm{P}=1.000$ \\
\hline & & Group C & $\mathrm{P}=0.993$ \\
\hline & \multirow[t]{2}{*}{ Group B } & Group A & $\mathrm{P}=1.000$ \\
\hline & & Group C & $\mathrm{P}=0.995$ \\
\hline & \multirow{2}{*}{ Group C } & Group A & $\mathrm{P}=0.993$ \\
\hline & & Group B & $\mathrm{P}=0.995$ \\
\hline
\end{tabular}

\section{Discussion}

A study was conducted by Mhatre BS and Singh YL et.al in 2013 on a title named 'Which is the better method to improve perceived hamstrings tightness, Exercises targeting neural tissue mobility or exercises targeting hamstrings muscle extensibility?' 56 female students with perceived hamstring tightness were recruited in this study. The study design was two group pretest posttest design. Systematic random sampling technique was used to collect the data. Subjects were randomly allocated into two groups. Treatment received by group A to improve neural mobility was Mulligan's Bent Leg Raise (BLR) technique followed by Two Leg Rotation technique (TLRT). To improve hamstrings muscle flexibility passive hamstrings stretching technique was received by Group B. knee flexion angle during Active Knee Extension test and Slump test were the outcome measures. There was statistically significant improvement observed in knee flexion angle for both tests in both groups. Inter group comparison showed that slump test was significantly improved in group which received neural tissue mobility exercises. Hence it was concluded that exercises which target neural tissue mobility are more effective as compared to exercises targeting hamstrings stretching in treating perceived hamstrings tightness [7]. The results were supported by the current study done by researcher. The study was randomized control trial, 39 females and 25 males were included in the study. Group A received Mulligan bent leg raise technique, Group B received Mulligan two legs rotation technique and group $\mathrm{C}$ received straight leg raise with traction technique. NPRS was significantly reduced and SLR was significantly improved in all 3 groups with $\mathrm{p}$ value $\mathrm{P}$ $<0.01$.

A study was conducted by Pratishtha K and Jagga V in 2012 with the title. Effect of Mulligan stretching techniques Traction Straight Leg Raise (TSLR) and Bend Leg Raise (BLR) on biceps femoris muscle and pelvic rotation by using surface EMG and bubble inclinometer. This study was aimed to investigate and compare the effectiveness of two Mulligan techniques (TSLR and BLR that is Traction Straight Leg Raise and Bend Leg Raise) in improving the biceps femoris muscle performance, flexibility and pelvic rotation. A total of 90 asymptomatic female subjects of 18 to 30 years ago were taken which were randomly divided into three groups, that is, two experimental (A and B) and one control group (C). The pelvic rotation ROM, passive straight leg raise ROM and EMG activity were checked in all three groups, after that the BLR, TSLR and Hamstring Self Stretch was provided to Group A, B and $C$ respectively. All the previous readings were checked again. TSLR stretching technique was found to decrease the EMG activity and increase the range of pelvic rotation and passive straight leg raise more than other two groups. It was concluded that Mulligan TSLR stretch is more effective than BLR stretch in improving biceps femoris muscle performance, flexibility and pelvic rotation [8]. All 3 Mulligan techniques were included in the current study. The present study concluded that SLR was significantly improved by all 3 Mulligan techniques (bent leg raise technique, two legs rotation technique and straight leg raise with traction technique) with $\mathrm{p}$ value $(p<0.01)$ but bent leg raise technique was more effective than TSLR and TLRT in improving hamstrings flexibility.

Previously a study was conducted by Hall T, Cacho A, McNee C, Riches J and Walsh J on Effects of the Mulligan traction straight leg raise technique on range of movement. It was noted that decreased range of SLR due to low back dysfunction and hamstring tightness was improved by using The Mulligan traction straight leg raise (SLR) technique. There were 26 normal subjects (mean age 26 years; 13 male) included in the study to evaluate the effects of this technique. Post intervention, the mean range of SLR increased significantly from $49.9^{\circ}$ to $63.2^{\circ}$, by $13.3^{\circ}$ or $27 \%$. Post intervention the main cause for increased SLR range was the hip flexion, showing an increased tolerance in hamstring muscle stretch [9]. the results 
were supported by the current study presenting that all 3 Mulligan (BLR, TLRT, and TSLR) were significantly effective in reducing NPRS and improving range of SLR after one session.

Hall T. et al conducted a study in 2006 titled Mulligan traction straight leg raise: A pilot study to investigate effects on range of motion in patients with low back pain. The study was conducted to evaluate the immediate effects of Mulligan traction straight leg raise technique (TSLR) on range of straight leg raise (SLR) in low back pain subjects, 19 subjects with low back pain and unilateral limited SLR were recruited in study. SLR, Hip flexion and posterior pelvic rotation were measured on $1^{\text {st }}$ visit by goniometer. Range of SLR and hip flexion as compared to pelvic rotation were significantly increased post intervention with a $\mathrm{p}$-value $\mathrm{p}<0.001$. it was concluded that Mulligan TSLR technique was preliminary evident in reduction of low back pain [10]. It was supported by the observations made in the current study representing SLR was significantly improved in subjects by using Mulligan techniques, post intervention with $\mathrm{p}$ - value $\mathrm{p}<0.01$.

\section{Conclusion}

It was concluded that all Mulligan techniques were effective in improving pain score and straight leg raise range of motion but bent leg raise technique was most effective among all.

\section{References}

1. Weerasekara I, Kumari I, Weerarathna N, Withanage C, Wanniarachchi C, et al. (2013) The prevalence of hamstring tightness among the male athletes of University of Peradeniya in 2010, Sri Lanka. Int J Phys Med Rehab 1(01).

\section{ISSN: 2574-1241}

DOI: 10.26717/BJSTR.2021.33.005417

Mubin Mustafa Kiyani. Biomed J Sci \& Tech Res

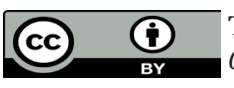

This work is licensed under Creative Commons Attribution 4.0 License

Submission Link: https://biomedres.us/submit-manuscript.php
2. Mhatre BS, Singh YL, Tembhekar JY, Mehta A (2013) Which is the better method to improve "perceived hamstrings tightness"-Exercises targeting neural tissue mobility or exercises targeting hamstrings muscle extensibility? International journal of osteopathic medicine 16(3): 153-162.

3. Radwan A, Bigney KA, Buonomo HN, Jarmak MW, Moats SM, et al. (2015) Evaluation of intra-subject difference in hamstring flexibility in patients with low back pain: An exploratory study. Journal of back and musculoskeletal rehabilitation 28(1): 61-66.

4. Draper DO, Castro JL, Feland B, Schulthies S, Eggett D, et al. (2004) Shortwave diathermy and prolonged stretching increase hamstring flexibility more than prolonged stretching alone. Journal of orthopaedic \& sports physical therapy 34(1): 13-20.

5. Fasen JM, O'connor AM, Schwartz SL, Watson JO, Plastaras CT, et al. (2009) A randomized controlled trial of hamstring stretching: comparison of four techniques. The Journal of Strength \& Conditioning Research 23(2): 660-667.

6. Puentedura EJ, Huijbregts PA, Celeste S, Edwards D In A, Landers MR, et al. (2011) Immediate effects of quantified hamstring stretching: holdrelax proprioceptive neuromuscular facilitation versus static stretching. Physical Therapy in Sport 12(3): 122-126.

7. Exelby L (2002) The Mulligan concept: its application in the management of spinal conditions. Manual therapy 7(2): 64-70.

8. Pratishtha K, Jagga V (2012) Effect of Mulligan stretching techniques (TSLR AND BLR) on biceps femoris muscle and pelvic rotation by using surface EMG and bubble inclinometer respectively. Journal of Exercise Science and Physiotherapy 8(1): 39-42.

9. Hall T, Cacho A, McNee C, Riches J, Walsh J, et al. (2001) Effects of the Mulligan traction straight leg raise technique on range of movement. Journal of Manual \& Manipulative Therapy 9(3): 128-133.

10. Hall T, Beyerlein C, Hansson U, Lim HT, Odermark M, et al. (2006) Mulligan traction straight leg raise: A pilot study to investigate effects on range of motion in patients with low back pain. Journal of Manual \& Manipulative Therapy 14(2): 95-100.

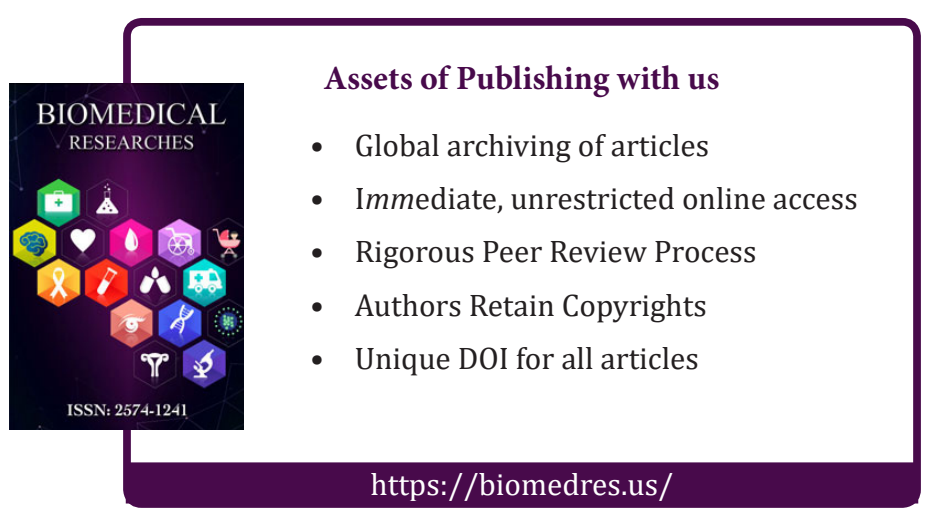

